\title{
Maintenance Management and Operational Support as Services in Reconfigurable Manufacturing Systems
}

\author{
Luis Ribeiro*, José Barata* \\ Paulo Leitão**, Neslon Silvério* \\ *New University of Lisbon, Monte de Caparica, Portugal \\ $\{l d r, j a b\} @$ uninova.pt,nss18303@fct.unl.pt \\ **Instituto Politécnico de Bragança, Bragança, Portugal \\ pleitao@ipb.pt
}

\begin{abstract}
Emergent architectures and paradigms targeting reconfigurable manufacturing systems increasingly rely on intelligent models to maximize the robustness and responsiveness of modern installations. Although intelligent behaviour significantly minimizes the occurrence of faults and breakdowns it does not exclude them nor can prevent equipment's normal wear. Adequate maintenance is fundamental to extend equipments' life cycle. It is of major importance the ability of each intelligent device to take an active role in maintenance support. This paper proposes a maintenance architecture supporting maintenance teams' management and offering contextualized operational support. All the functionalities hosted by the architecture are offered to the remaining system as network services. Any intelligent module, implementing the services' interface, can report diagnostic, prognostic and maintenance recommendations that enable the core of the platform to decide on the best course of action.
\end{abstract}

Keywords: Maintenance, Intelligent Manufacturing Systems, Adaptative Systems, Agents.

\section{INTRODUCTION}

To withstand current and future socio-economic pressures enterprises increasingly seek innovative forms of networked organization. The shop floor is one of the most critical assets in this restructuring process.

Recent developments in networked information technologies present major opportunities for integrating the shop floor as an active entity and a motor of competitiveness (Stamatis, Colombo et al. 2007). Additionally there is a significant ongoing effort for preparing the next generation of assembly systems which is denoted by the emergence of industrial paradigms: bionic manufacturing systems (BMS) (Ueda 1992), holonic manufacturing systems (HMS) (Babiceanu and Chen 2006), reconfigurable manufacturing systems (RMS) (Koren, Heisel et al. 1999), evolvable assembly systems (EAS) (Onori 2002) and evolvable production systems (EPS) (Barata, Frei et al. 2007).

These modern control approaches have set the theoretical background and foresee modular and distributed architectures where local intelligence and interaction play a significant role.

Local intelligence is fundamental to ensure that the system exhibits a coherent and desirable behaviour. However it does not exclude, although it minimizes, occurrence of faults and breakdowns. In (Barata, Ribeiro et al. 2007; Ribeiro 2007) the rationale for adopting self-diagnosis and, under controlled conditions, self-healing in the context of EAS/EPS has been detailed. The potential profit emerging from these mechanisms can be further extended if their input positively influences equipment's maintenance cycles.

Maintenance has traditionally been regarded as necessity. Nevertheless it represents a share of $15 \%$ to $60 \%$ of the total production costs (Mobley 2002) depending on the goods produced.

The previous discussion enforces (Tsang 2002) where the drivers for increased performance requirements in maintenance are identified as: emerging trends of operational strategies, toughening societal expectations, technological changes, changes in the people and organizational systems.

As the role of maintenance has a strategic pillar of enterprise competitiveness is acknowledged the notion of emaintenance is consolidating as a reference paradigm. The research focus has been set on the development of diagnostic and prognostic techniques and methods and the integration of their results with business logic tools. The purpose of the present paper is proposing an architecture that provides local maintenance operational support, which has been relatively unexplored in the literature, specifically issuing predictive, preventive and repair (critical) maintenance recommendations, organizing the best possible maintenance teams accordingly and assisting specialized technicians during the maintenance process. All the functionalities are offered as network services to foster integration and plugability while all the reasoning is agent-based. The subsequent sections are organized as follows: section two overviews related work focusing on e-maintenance; section three depicts the proposed architecture; section four briefly details the implementation work using the JADE Web 
Services Integration Gateway (WSIG) infrastructure; section five presents some preliminary conclusions.

\section{RELATED WORK}

The concept of E-Maintenance was introduced in recent literature (since 2000) and despite the lack of consensus in a common definition (Iung, Levrat et al. 2007) most of the works cover the following issues: multilevel network integration of maintenance activities and related applications and attaining near zero device downtime.

Moreover E-maintenance advocates the incorporation of maintenance in product life cycle. In (Takata, Kimura et al. 2004) the concept of life cycle maintenance was introduced to stress the importance of maintaining the products in an acceptable functional level during their lifecycle while continuously improving them as well as maintenance techniques so that existing products can adapt to changes in the operational environment.

The concept of E-Maintenance has been driving several projects worldwide.

One of the most active research centres in maintenance is the intelligent maintenance systems (IMS 2007) (IMS) group that seeks the achievement of near-zero breakdown performance. The IMS developed the Watchdog Agent ${ }^{\mathrm{TM}}$ (Lee, Ni et al. 2006) to implement CBM. This system collects data from sensors monitoring critical processes and matches it against known sensor signatures to detect process degradation.

Moreover prognosis is supported by applying trending and statistical modelling over the process signatures while diagnosis is based in the memorization of relevant signature patterns of past events. Additionally the Watchdog Agent ${ }^{\mathrm{TM}}$ is integrated with the IMS Device-to-Business (D2B ${ }^{\mathrm{TM}}$ ) (Huang, Xi et al. 2005) platform that provides a link between the shop floor and e-business.

In (Han and Yang 2006) a maintenance framework, based in the concept of maintenance centre, is presented. Each maintenance centre is divided in: Fundamental research group - research in recent technologies and simulation analysis; Enterprise group - provide information flow between associated enterprises; Expert group - grant support to maintenance system research and improve and optimize the research results; Industry case history collection group collect knowledge from the industrial environment to feed a case database to be used later in case based reasoning for diagnosis, risk assessment, help desk, decision support and maintenance strategy.

Moreover the framework relies in local maintenance centres at plant level that coordinate the following activities: continuous assessment of equipment health, maintenance and repair operation process management and comprehensive data representation and synthesis. In this context the local maintenance system works as a test platform for the results derived from the maintenance centre.

The problem-oriented multi-agent-based E-service system (POMAES) proposed in (Yu, Iung et al. 2003) explores co- ordination, co-operation and negotiation in multiagent systems to solve problems in the industrial context. This raises the notion of collaborative maintenance. In this context a prototype implementation was applied to control a water valve. In this setup three experts were considered: Production management expert - controls the water volume; Maintenance expert - optimizes the valve and the platform availability by minimizing direct and indirect costs related to maintenance; Control expert - ensures the behaviour of the installation is in accordance to the production strategy.

Through interaction a balanced compromise is achieved that maximizes availability keeping maintenance costs controlled.

Other maintenance platforms include PROTEUS (Bangemann, Rebeuf et al. 2006) that focus on the integration platform rather than on the development of dedicated maintenance tools. From an architectural point of view PROTEUS denotes a star like shape being the central point occupied by the central service application (CSA) that provides integration oriented services. Additionally the platform relies in intelligent core adapters (ICA) to provide standardized interface transformers for the peripheral applications and functional core applications (FCA) to implement supplementary functions needed by the global service requirements and not provided by the platform tools.

A complete review on the concept of e-maintenance, major achievements and current research is provided in (Muller, Marquez et al. 2007).

The work later detailed has some contact points with (Garcia, Guyennet et al. 2004) in the sense that the presented maintenance architecture facilitates the establishment of remote sessions for collaborative maintenance. However, the architecture proposed in the next chapter focus entirely in maintenance management aspects including, among other things, a strong emphasis on the automatic selection of the best local maintenance teams and provides contextualized onsite support with the level of detail adequate to each member of the team.

\section{ARCHITECTURE}

\subsection{Architecture Overview}

Under the framework of RMS it is assumed that each active module is autonomous, intelligent and has its own computing hardware. Each module performs self-monitoring/diagnosis and is able to use that information to issue predictive maintenance alarms in addition to time-based (according to the manufacturer's recommendation) preventive maintenance alarms. Furthermore all its related documentation (technical manuals, blueprints, repair/maintenance procedures, etc) is stored locally.

The intelligent modules connect to the maintenance subscribing and invoking its services reporting data when necessary as detailed in Figure 1. 


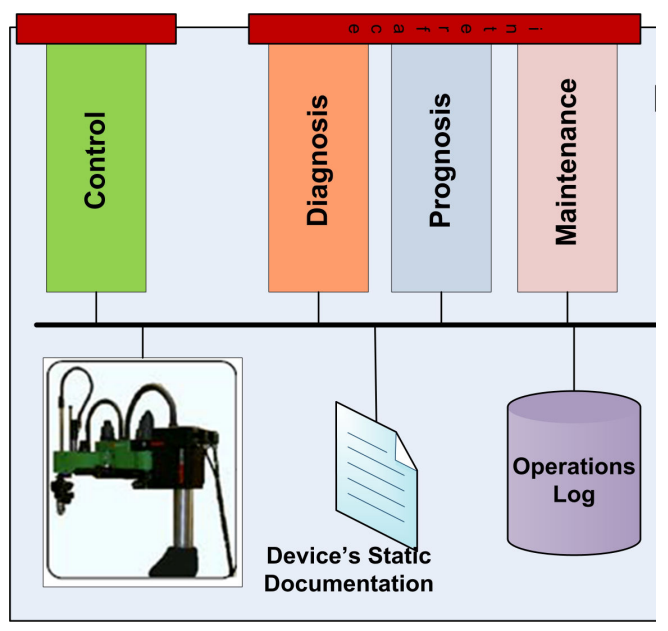

Fig. 1. Interaction at module level.

It is assumed that the diagnosis/prognosis and maintenance functions, at module level, are according to the computational power available. In this context the present architecture does not induce any constraint in that matter relying on the service's interfaces to harmonize all the incoming and outgoing information.

Each device notifies the core platform of relevant events (maintenance, diagnosis and prognosis) upon occurrence. Furthermore heart beat notification must be present to ensure that the core platform is aware of the presence of the device and otherwise.

The mandatory functionalities present at device level are kept at a minimum level to maximize plugability and integration.

The core of the architecture processes and merges all the input data reported. An overview of the architecture is depicted in. Figure 2.

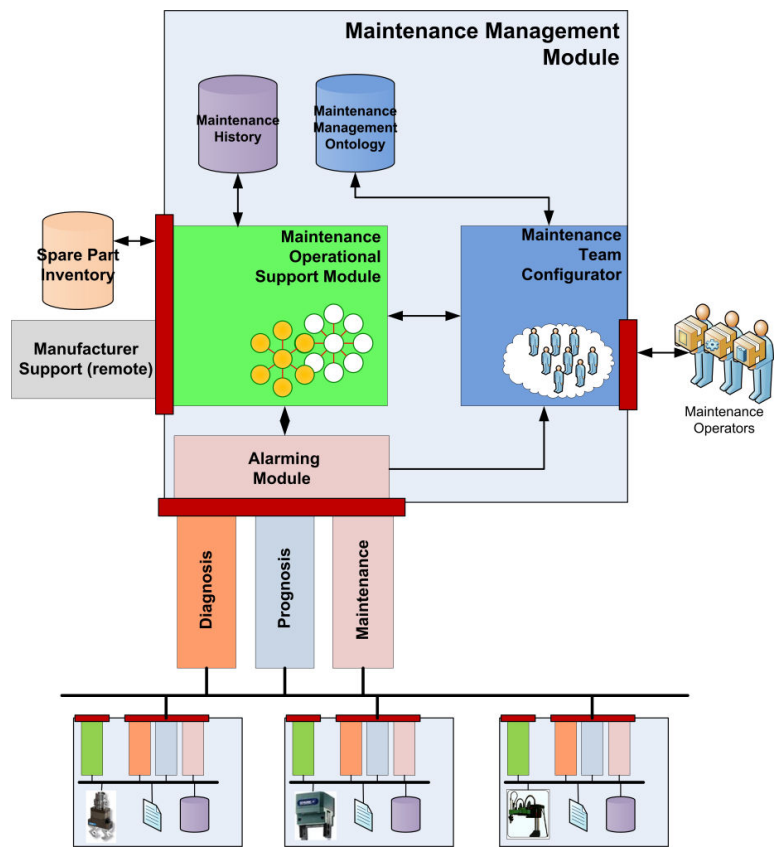

Fig. 2. Architecture overview.
The maintenance module comprises several blocks of different nature. External data, including prognostic and diagnostic information, spare part inventory, etc, is accessed through the use of interfaces for harmonization and plugability purposes.

The Alarming Module (AM) processes the information incoming from the intelligent modules registered in the system and stores it until it is consumed by the Maintenance Team Configurator (MTC) and to the Maintenance Operational Support Modules (MOSM). Furthermore the AM notifies the devices that have issued through their maintenance interface according to the info conveyed by the MOSM.

The Maintenance History Database (MHD) stores all the reported events in maintenance logs. Each record includes the date and nature of the event as well as the action taken and the technician's report (including the main decisions taken if any are not in accordance with the recommended procedures).

The Maintenance Management Ontology (MMO) maps the main concepts and relations supporting the system, including: characterization of maintenance actors and their skills, rules for role assignment during and maintenance team formation, clearance and trust levels, etc.

The Maintenance Operational Support Module (MOSM), depicted in Figure 3, supports data mining and dedicated operational support.

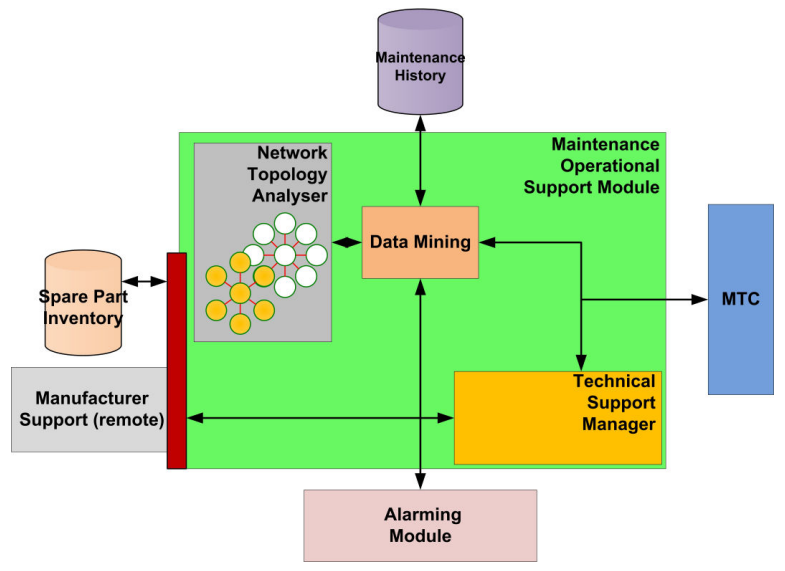

Fig. 3. Maintenance Operational Support Module.

The data mining functionalities of the MOSM include correlation of the topology of the network with the faults reported and the maintenance cycles of the equipment reported in the MHD. The generated knowledge enables:

- Implementing system-wise condition based and predictive maintenance.

- Identifying correlations and patterns in the event reported. 
- Recommending and negotiating with the MTC technicians for maintenance tasks based on their experience.

The Technical Support Manager (TSM) abstracts each member of a maintenance team as a software entity and enables them to seamlessly interact with the remaining system, through that entity, according to the MTC recommendations. The level of support provided to the technician will be later detailed in the use case section.

The Maintenance Team Configurator (MTC) maintains a pool of the available maintenance technicians, their skills and the pending maintenance/repair tasks (Figure 4).

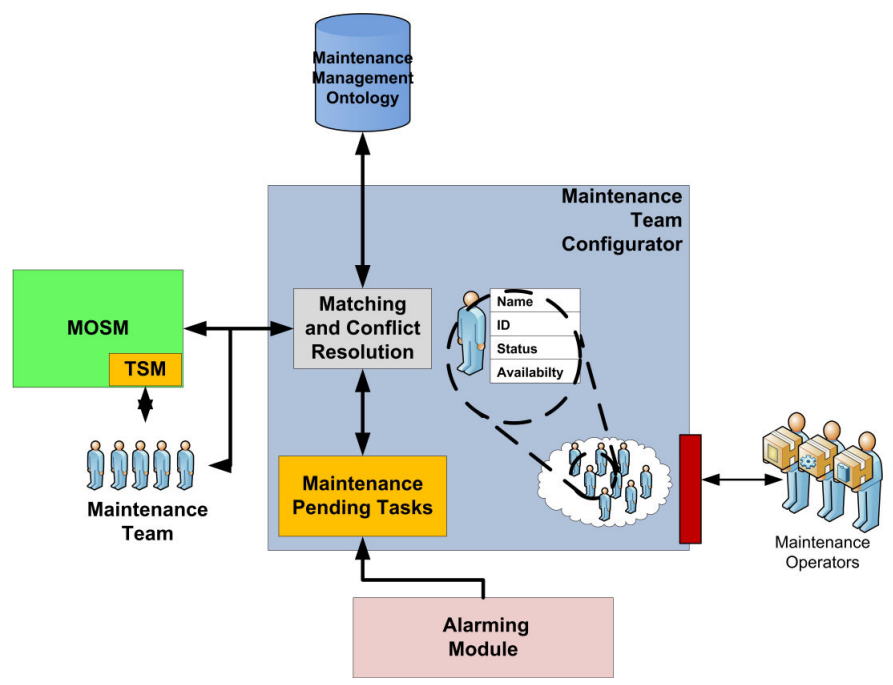

Fig. 4. Maintenance Team Configurator

Whenever a recommendation or a repair alarm is received the MOSM suggests a team based on the maintenance history of the failing modules. The MTC will match this information against the knowledge in the MMO and the availability of the technicians. Conflicts in the matching are solved by the rules of the MMO until the best possible team is constituted. Team formation comprises:

- Evaluating the urgency of the pending maintenance tasks.

- Assigning and negotiating each participant's roles.

- Notifying the participants of the nature and location of task.

- Handing over the control to the MOSM entities in the TSM recommending on the adequate support.

The last bullet is worth an extra remark. The MTC may not be able to gather the optimal team. According to the urgency of the task a less specialized member can be present. The MTC may recommend the TSM to establish a remote session within the Manufacturer's Support Centre.

The module can alternatively postpone the execution of maintenance by acknowledging that a certain technician will be available within a reasonable time interval.

\subsection{Use Cases}

As mentioned the maintenance technicians interact mainly with the MTC and the MOSM. It is assumed that this interaction happens through any handheld computing device.

Figure 5 details basic interactions with the MTC.

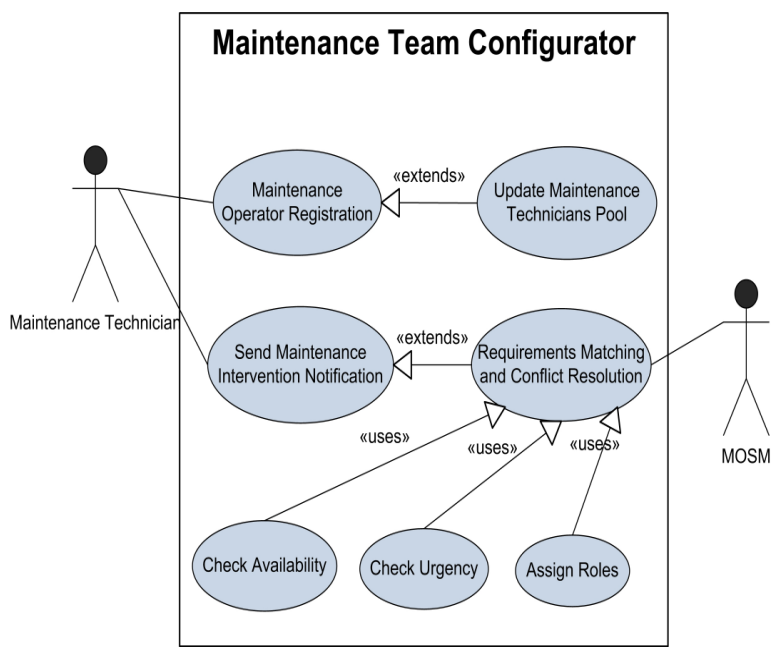

Fig. 5. Registering and receiving notifications

Whenever a maintenance technician is active he registers within the Maintenance Module through the MTC.

The MTC will, from that point on, until the unregister procedure, consider that technician has a candidate to incorporate any maintenance team.

Whenever a maintenance task is scheduled the operator will receive in his handheld device a notification informing him that he has been allocated to a maintenance team and is prompted to confirm his participation in the process.

This first notification includes the location and nature of the equipment that will be maintained as well as authentication codes that identify him and allow him to use his MOSM entity.

Upon authenticating in the MOSM the technician receives instructions on preliminary actions that he must execute before arriving at the maintenance scene. This list may include collecting spare parts or specific equipment at other destinations also detailed in the notification.

When a team is allocated and its presence on spot is confirmed each participant receives contextualized information on how he should proceed.

According to the expertise, clearance level and the MTC recommendations the MOSM may:

- Allow the operator to override some instructions and alter the maintenance procedures.

- Provide on demand information concerning the device. 
- Support a remote session for collaborative interaction with the manufacturer's maintenance centre.

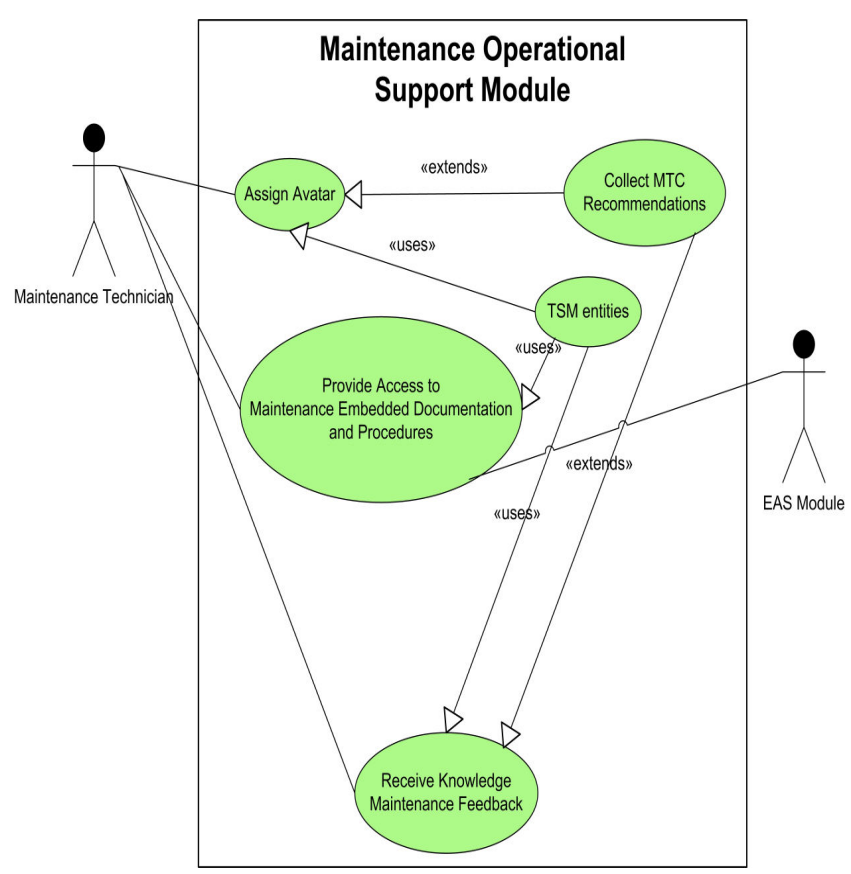

Fig. 6. MOSM Operational Support

These interactions are possible using the individual entities attributed by the MOSM as shown in Figure 6.

\section{IMPLEMENTATION}

In the previous section the main aspects of the architecture functioning have been described. From an implementation point of view the envisioned system imposes some constraints in the supporting IT infrastructure:

- The intelligent modules connected to the maintenance system constitute a distributed environment of potentially undetermined size.

- The nature of the interactions between all the actors (Intelligent modules, Maintenance Module, Maintenance Technicians, etc) is asynchronous and several actions may occur simultaneously.

- Some actions might have to be prioritized.

- The system relies in data mining. Knowledge and information must be both processed by humans and machines.

To overcome or minimize this challenges the implementation of the present architecture is supported by the JADE Web Services Integration Gateway (WSIG).

The WSIG platform allows the agent functionalities to be expressed as a regular web service (WS) that can be published, discovered and implemented using the conventional WS mechanisms.
The MOSM comprises two fixed agents: the Data Mining Agent (DMA) and the Technical Support Manager Agent (TSMA). The TSMA creates and manages one Avatar Agent (AA) per maintenance team member to track their actions.

This distribution is justified by the fact that the DMA must do background data mining work while being able to support the MTC. As several technicians can input the TSM asynchronously each one is assigned a AA. For coherence all the inputs from the different teams are managed by the TSMA.

The MTC is also composed by two agents: the Matching and Conflict Resolution Agent (MCRA) and the Maintenance Pending Task Agent (MPTA). The latter prioritizes maintenance tasks and the first assigns the maintenance teams. This distribution will enhance the reactivity of the system to severe failures.

The Alarming Module Agent (AMA) interfaces the intelligent modules with the Maintenance Module. To improve plug-ability in heterogeneous environments the AMA manages several Interface Agents (IA) that harmonize information from different sources.

Figure 7 shows the WSDL description considered for reporting diagnostic information.

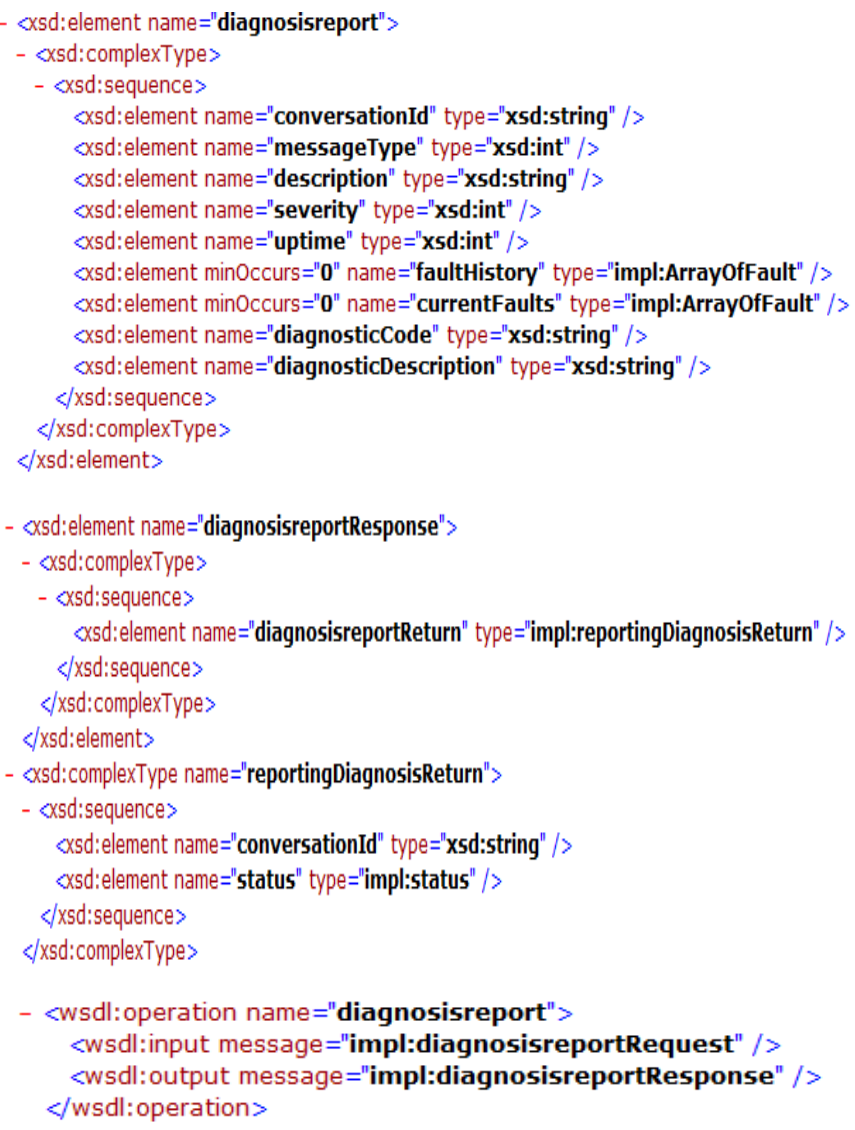

Fig.7. WSDL description of a diagnostic report

As shown in Figure 7 after diagnosing the fault the intelligent module forwards complete diagnostic information including 
its complete fault history and the current faults that lead to the present diagnosis.

\section{CONCLUSIONS}

In the present work an architecture is proposed to support local maintenance management. Rather than specifying a complete solution for the maintenance problem a selfcontained and pluggable solution is advocated. In particular the architecture targets systems that are compositions of intelligent modules that naturally exhibit autonomy, intelligence and possess own processing power. However any similar system can be considered. Its main contributions are:

The possibility of optimizing maintenance teams according to the maintenance history, skills and availability of the technicians as well as the urgency of the occurrence.

Assisting in maintenance operations defining roles, coordinating the activities and accepting controlled feedback.

Extracting new knowledge from the maintenance historical data to influence future actions as well as assessing the impact of performing maintenance in correlated equipment.

WSIG is used as a base platform as it allows exposing agent functionalities as web services that can be used in any network using existing and widely spread web standards.

It is the authors' belief that this modular and hybrid (service and agent oriented) approach to maintenance systems can be easily integrated in any installation and accompany the dynamics of modern reconfigurable systems.

\section{ACKNOWLEDGEMENTS}

This work was partially supported by POSC/FEDER.

\section{REFERENCES}

Babiceanu, R. and F. Chen (2006). "Development and applications of holonic manufacturing systems: a survey." Journal of Intelligent Manufacturing, 17: 111-131.

Bangemann, T., X. Rebeuf, et al. (2006). "PROTEUS Creating distributed maintenance systems through a integration platform." Computers in Industry 75(6): 539-551.

Barata, J., R. Frei, et al. (2007). Evolvable Production Systems Context and Implications. International Symposium on Industrial Informatics. Vigo, IEEE.

Barata, J., L. Ribeiro, et al. (2007). Diagnosis on Evolvable Production Systems. International Symposium on Industrial Electronics. Vigo, IEEE.

Garcia, E., H. Guyennet, et al. (2004). "A new industrial cooperative tele-maintenance platform." Computers and Industrial Engineering 46: 851-864.

Han, T. and B.-S. Yang (2006). "Development of an emaintenance system integrating advanced techniques." Computers in Industry 57: 569-580.

Huang, R., L. Xi, et al. (2005). "The framework impact and commercial prospect of a new maintenance system: intelligent maintenance system." Production Planning and Control 16(7): 652-664.

IMS. (2007). "Center for Intelligent Maintenance System." from http://www.imscenter.net/.

Iung, B., E. Levrat, et al. (2007). E-Maintenance: Principles, Review and Conceptual Framework. Cost Effective Automation in Networked Product Development and Manufacturing. Monterrey, N.L. México, .

Koren, Y., U. Heisel, et al. (1999). "Reconfigurable Manufacturing Systems." CIRP Annals Manufacturing Technology 48(2): 527-540.

Lee, J., J. Ni, et al. (2006). "Intelligent prognostics tools and e-maintenance." Computers in Industry 57(6): 476 489.

Mobley, R. K. (2002). An Introduction to Predictive Maintenance, Butterworth-Heinemann.

Muller, A., A. C. Marquez, et al. (2007). "On the concept of e-maintenance : Review and current research." Reliability Engineering and System Safety (article in press doi:10.1016/j.ress.2007.08.006).

Onori, M. (2002). Evolvable Assembly Systems - A New Paradigm? 33rd International Symposium on Robotics Stockholm.

Ribeiro, L. (2007). A Diagnostic Infrastructure for Manufacturing Systems. Electrical and Computer Science Engineering. Lisbon, New University of Lisbon. MSC: 121.

Stamatis, K., A. W. Colombo, et al. (2007). Towards Serviceoriented Smart Items in Industrial Environments. International Newsletter on Micro-Nano Integration - MST News, VDI/VDE-IT: 11-12.

Takata, S., F. Kimura, et al. (2004). "Maintenance: Changing Role in Life Cycle Management." Annals of CIRP 53(2): 634-655.

Tsang, A. H. C. (2002). "Strategic dimensions of maintenance management." Journal of Quality in Maintenance Engineering 8(1): 7-39.

Ueda, K. (1992). A concept for bionic manufacturing systems based on DNA-type information. PROLAMAT. Tokyo, IFIP.

Yu, R., B. Iung, et al. (2003). "A multi-agents e-maintenance system with case-based reasoning support." Engineering applications of artificial intelligence 16: 321-333. 